One of the only criticisms I would have of the book is the fact that there are very limited colour photographs and diagrams, which can make the pages appear a little dull.

I can see why previous editions of this book have been so popular. It is easy to dip in and out of, so knowledge on particular topics can be easily refreshed. The clear explanations make a potentially arduous topic to revise an interesting read. A must have for undergraduates and GDPs alike!

L. Ridsdale

\section{IMPLANT SITE DEVELOPMENT}

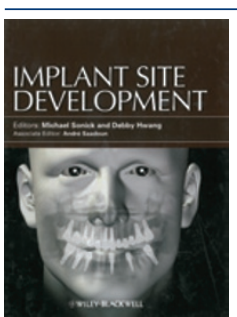

M. Sonick, D. Hwang (eds)

Wiley-Blackwell

price $€ 117.00$; pp 456

ISBN 9780813825120

Implant site development refers to a variety of procedures aimed at augmenting an edentulous ridge to optimise implant positioning for excellent prosthesis aesthetics and function. From the outset, the editors make abundantly clear implant placement and augmentation of the site should be prosthetically driven.

The text begins with an overview of bone metabolism and healing, which is concise and focuses on factors underpinning clinical decisions. Following this, a refreshingly pragmatic, albeit flamboyantly written section by the editors on their treatment philosophies include 'not to rush treatment' and 'aesthetic implant restorations are fraught with risk'.

Anatomy of the jaws was covered extensively in two chapters illustrated with excellent quality images form cone beam computer technology (CBCT). Numerous clinical cases illustrated virtual implant placement highlighting shortcom-

ings of alveolar dimensions and critical anatomical structures that require careful attention. Well-appointed, clearly labelled diagrams explaining key dimensions for implant positioning help the reader relate to the extensive and scrupulous intraoperative photographs during treatment.

This nicely sets the scene to detail the options of implant site development.
Ridge augmentation procedures such as pre-extraction orthodontics, socket preservation, and pre- and perioperative implant placement grafting are initially covered. Pleasingly the authors have neatly categorised the plethora of currently available grafting materials into visually attractive tables and flow charts. Block bone grafting from extra and intraoral donor sites and maxillary sinus augmentation procedures follow, but with progressively fewer illustrated cases. Distraction osteogenesis and soft tissue augmentation completed the list of surgical options. Unfortunately, provisionalisation and prosthetic considerations were only briefly discussed.

The authors have gone to great lengths to cover all potential clinical techniques, available materials, potential complications and future developments associated with implant site development. This has led to slight repetition and pushing the boundaries of what is achievable; some techniques mentioned require extensive clinical experience and knowledge beyond this text. As an overview for clinicians with an interest to begin implant treatment it is on the whole well referenced, utilising excellent high quality illustrations making an informative and pleasurable read.

G. Calvert

\section{STEM CELLS IN CRANIOFACIAL DEVELOPMENT AND REGENERATION}

\begin{tabular}{cl}
\hline STEM CELLS & G. T. J. Huang, I. Thesleff (eds) \\
Wiley-Blackwell \\
In CRANIOFACIAL \\
DEVELOPMENT Ano \\
REGENERATION & price E100.00; pp 584 \\
& ISBN 9781118279236 \\
& \\
\hline &
\end{tabular}

In recent years, major advances have been made in stem cell biology and in tissue engineering technology. As a result of this rapidly evolving niche, this text focuses on the potential use of tissue specific stem cells in bioengineering of craniofacial tissues and organs. It is an introduction to stem cells with an emphasis on their role in craniofacial development. This text is divided into three sections and contains 28 well written chapters. Filled with superb, full-colour photographs and step-by-step, illustrated procedures, this book is the definitive source on all aspects of stem cells in craniofacial development.

Stem cells have the unique ability to repair and replenish adult tissues and are now being utilised in cutting-edge medical therapies. The field of stem cell biology focuses on the full spectrum of cell behaviour from the stem cell state to the fully differentiated condition. With contributions from a coterie of renowned experts in the field of craniofacial research Stem cells in craniofacial development and regeneration should become the vade mecum of aspiring researchers, developmental biologists, tissue engineers and dentists. This comprehensive text can also be utilised effectively by oral and maxillofacial surgeons and colleagues in related fields. This edition reflects the latest trends, concepts and innovations in the field of stem cell regeneration.

The first section of the book is divided into nine chapters discussing the embryogenesis, morphogenesis and regeneration of craniofacial tissues and organs. Cranial neural crest cells, temporomandibular joint and craniofacial muscle development are high-lighted. In addition, paragraphs are devoted to understanding the formation, proliferation, migration and differentiation of neural crest cells. Various contributors explain how craniofacial anomalies occur and discuss craniosynostosis and Treacher Collins Syndrome. In order to develop therapeutic strategies for minimising or preventing craniofacial anomalies it is imperative to understand the normal events that induce neural crest cells and influence their differentiation during embryogenesis.

The authors consolidate prior knowledge of stem cells in the second section of the book where they elaborate on the concept of induced pluripotent stem cells. The third section of the book focuses on stem cell mediated craniofacial tissue bioengineering. It summarises the use of scaffolds, growth factors and stem cells in engineered tissue regeneration. Chapters reinforce periodontal bioengineering strategies including the present status and some developing trends for the future. 
This text offers complete coverage of stem cells in craniofacial development and regeneration highlighted by an unparalleled collection of outstanding, full-colour photographs and highly detailed drawings that clarify procedures. This book is a welcome addition to any library and is an essential reference tool for those engaged in stem cell biology.

\section{Dunphy}

\section{BUSINESS BASICS FOR DENTISTS}

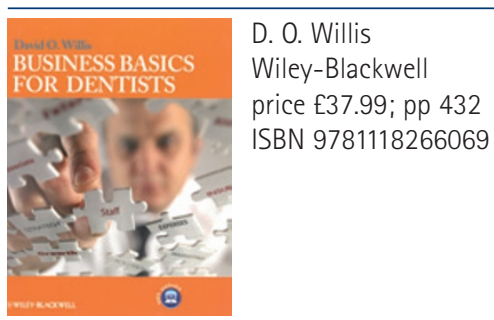

Business basics for dentists provides an interesting overview on managing a successful dental practice and aims to add to the business knowledge and skills of the dentist. David 0. Willis, in his preface, notes that this book is primarily for dental students about to graduate or recent graduates who have been out of dental school for five years or less - I would agree. This is a book written to educate the new dentist who is least prepared in practice management. The concepts discussed in the chapters are basic and form a foundation for understanding the financial aspects of the field.

The book is divided into four easyto-read sections. It is designed for convenient access with clearly defined chapters to make it easy to refer to by the busy practitioner. The wellstructured text is complemented with a rich variety of text boxes, summarised tables and graphs. The text in each chapter is based on mainstream concepts tempered with practical suggestions gleaned from the author's experience in working with new and young practitioners.

Chapter eight is primarily based on American laws and regulatory bodies that are not always applicable to dental practice in other parts of the world, but it is not within the remit of this book to do so.

This book contains a particularly interesting chapter on career planning that many dental students and new graduates will find insightful and thought-provoking. The book aims to provide solutions for many management problems that I could relate to as a practising clinician. I thoroughly enjoyed reading this book and learnt a lot of tips that are useful in daily practice.

In summary, a well-structured and written reference book accumulating knowledge of a very experienced clinician enables this book to be of relevance to the newly qualified dentist and a tool to reinforce well-known concepts with stimulating ideas to the well-established dentist.

S. Abdul-Razzak

\section{MASTER DENTISTRY. VOLUME 2: RESTORATIVE DENTISTRY, PAEDIATRIC DENTISTRY AND ORTHODONTICS, 3RD EDITION}

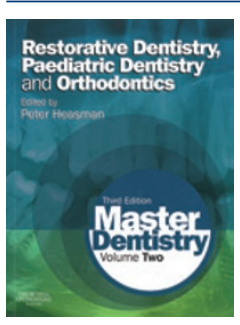

P. Heasman (ed) Churchill Livingstone price $€ 29.99$; pp 436 ISBN 9780702045974

Revision is a uniquely tough and sometimes lonely place: it is you, 'the books' and a looming deadline; one which gets more ominous as every hour and day passes. The key to success during this time is to concentrate on improving your weaknesses whilst maintaining your strengths - one element to ensuring you get this balance right is the use of quality revision guides such as Master dentistry, Volume 2.

This text manages to accomplish the seemingly impossible task of condensing the three large specialties of restorative dentistry, paediatric dentistry and orthodontics into a single compact tome. It achieves this feat by covering the basic undergraduate curriculum without labouring on contentious issues or excessively fine detail. The book has been designed in such a way that once you are roughly familiar with the text you can easily reference back to chapters and topics, each of which sets out key learning objectives at the beginning that helps to keep your revision efficient and relevant to your learning needs. Each of the 12 chapters cover the essential topics of describing health, clinical assessment, pathology and clinical techniques for treatment, the text is complemented by quality diagrams, pictures and excellent tables.

At the end of each chapter, the inclusion of self-assessments based on popular exam formats is particularly successful and useful as it allows the reader to objectively test their knowledge whilst improving exam technique. The editor's mindfulness of the target audience of this book is further demonstrated by a five-page preface discussing how to get the best out of the book for your revision and how best to prepare for popular exam types.

As a reader who is familiar with the second edition version during my own undergraduate revision sessions, I notice that the third edition is physically smaller in size, but with no reduction in the quantity or quality of the content. Furthermore, the reader can be reassured of the quality of the content as all of the contributing authors are renowned professors, consultants or experts in their field.

This books applicability to those taking postgraduate examinations such as the MJDF or MFDS examinations is enhanced by the inclusion of additional, unexpected, chapters including implantology, conscious sedation and law and ethics, which are also useful for those general dental practitioners who may be looking to gain a basic grounding before embarking on additional training in these fields.

P. Saini 